\title{
Green self-assembly synthesis of porous lignin-derived carbon quasi- nanosheets for high-performance supercapacitors
}

\author{
Fangbao Fu ${ }^{\mathrm{a}}$, Dongjie Yang ${ }^{\mathrm{a}}$, Wenli Zhang ${ }^{\mathrm{c}}$, Huan Wang ${ }^{\mathrm{a}, *}$, Xueqing Qiu ${ }^{\mathrm{a}, \mathrm{b}, *}$ \\ ${ }^{a}$ School of Chemistry and Chemical Engineering, Guangdong Provincial Engineering Research Center for Green Fine Chemicals, South China University of Technology, \\ 381 Wushan Road, Tianhe District, Guangzhou 510641, China \\ ${ }^{\mathrm{b}}$ State Key Lab of Pulp and Paper Engineering, South China University of Technology, Guangzhou 510641, PR China \\ ${ }^{\mathrm{c}}$ Materials Science and Engineering, King Abdullah University of Science and Technology (KAUST), Thuwal 23955-6900, Saudi Arabia
}

\section{H I G H L I G H T S}

- Industrial waste lignosulfonate is used to prepare high valued carbon materials.

- $\mathrm{LS} / \mathrm{ZnC}_{2} \mathrm{O}_{4}$ composite is fabricated in ethanol/water solution without any toxic material.

- PLC is prepared by Gas-exfoliation and In-situ templating carbonization technique.

- PLC with rational pore distribution, large surface area, and excellent conductivity.

- PLC supercapacitor shows great electrochemical performances in energy storage.

\section{A R T I C L E I N F O}

\section{Keywords:}

Porous lignin-derived carbon

Lignosulfonate

Self-assemble

In-situ carbonization

Supercapacitors
G R A P H I C A L A B S T R A C T

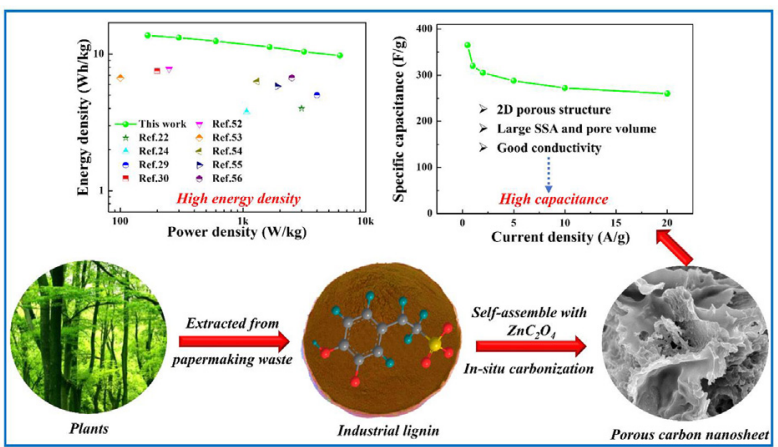

\begin{abstract}
A B S T R A C T
Two-dimensional porous carbon materials are very promising for energy storage/conversion due to their unique microstructure, reasonable pore structure and excellent electrochemical properties. A green and facile in-situ carbonization technique is innovated to prepare a unique porous lignin-derived carbon quasi-nanosheets (PLC) with rational pore distribution, large surface area, and excellent conductivity. The lignosulfonate/zinc oxalate composite was firstly self-assembled by hydrophobic bond of the amphiphilic structure in lignosulfonate using ethanol/water solvent without the need to use any toxic material, followed by co-pyrolysis at a high temperature with gas-exfoliation and in-situ templating of zinc oxalate. The resulting PLC exhibits a very high specific capacitance of $320 \mathrm{~F} / \mathrm{g}$ at $1.0 \mathrm{~A} / \mathrm{g}$ and long cycling stability (remains $93.5 \%$ after 10,000 cycles at $5.0 \mathrm{~A} / \mathrm{g}$ ). In addition, when assembling into symmetric supercapacitors in PVA/KOH gel electrolytes, PLC also shows a high specific capacitance of $274 \mathrm{~F} / \mathrm{g}$ at $0.5 \mathrm{~A} / \mathrm{g}$ with excellent rate capability and a high specific energy density $(9.75 \mathrm{~W} \mathrm{~h} / \mathrm{kg}$ at $6157.9 \mathrm{~W} / \mathrm{kg})$. These excellent electrochemical performances indicate that the as-prepared PLC should hold great promise for the energy storage devices, opening a new path for the preparation of advanced carbon electrode material and high-value-added utilization of biomass.
\end{abstract}

\footnotetext{
* Corresponding authors.

E-mail addresses: cewanghuan@scut.edu.cn (H. Wang), cexqqiu@scut.edu.cn (X. Qiu).
} 


\section{Introduction}

In recent years, supercapacitors, bridging the power gap between traditional capacitors and batteries, are currently attracting growing attention in the field of new energy storage devices owing to their ultrahigh power density, ultrafast charge-discharge rate, long cycle life, and environmentally friendly nature [1-7]. The electrode materials are usually considered as the key factor determining the performance of supercapacitors and carbon electrode materials are considered to be the most commercially available. To meet the demand of high-performance supercapacitor, various nanotextured carbon materials such as activated carbon, graphene, carbon nanofibers, carbon aerogels, and carbon nanotubes have been handily developed for supercapacitor electrode materials because of their tunable microstructure, superior electrochemical properties, and favorable charge transport [8-13]. As the representative of $2 \mathrm{D}$ carbon material, graphene possesses open $2 \mathrm{D}$ lamellar structure, which shortens the diffusion distance for ions and provides quick path for electron transport, thus leading to enhancement in electrochemical properties $[14,15]$. However, its practical application has been subject to high cost and short supply. This brings out great interest in seeking alternative low-cost porous carbon electrode materials.

Porous carbons are usually obtained by calcinating carbonaceous precursors in high temperature with the activation of strong corrosive agents, such as $\mathrm{KOH}, \mathrm{ZnCl}_{2}$, and $\mathrm{H}_{3} \mathrm{PO}_{4}[16,17]$. The acitivation processes are rather simple, but the obtained carbons are easily to aggregate, resulting in unordered pore structure and unsatisfied electrochemical performances. And the precursors are mainly non-renewable fossil or mineral resources which are unsustainable. Therefore, from a long-term perspective of sustainable development, seeking for renewable carbon source, as well as green preparation technique, is of great significance for application of carbon electrode.

Biomass (e.g., cellulose, lignin, chitin, chitosan, etc.) with the advantages of availability, abundance, renewability, and non-toxicity is regarded as an alternative carbon precursor and has been successfully converted into various types of nanoporous carbon recently [18,19]. As the only renewable aromatic polymer in natural plant, lignin is composed of guaiacyl, syringyl, and p-hydroxyphenyl propane structural units, occupying about $30 \%$ of organic carbon in biosphere [20]. The industrial lignin is the major by-product from pulping or biorefinery industry, and totals over 50 million tons annually. It can be classified into water-insoluble alkali lignin and enzymatic lignin, along with water-soluble lignosulfonate and the sulfonated product of alkali lignin. Unfortunately, only ca.10\% of industrial lignin is effectively utilized as dispersants or water reducers, and the rest is regarded as waste or burned as low-grade fuel [21]. Converting carbon-rich lignin into highvalued carbon materials would be a feasible way to address this issue, and it achieves the purpose of "waste into wealth".

Water-insoluble lignin shows seriously intermolecular aggregation, resulting in poor compatibility with activators, thus large amounts of strong corrosive reagents (e.g., $\mathrm{KOH}, \mathrm{ZnCl}_{2}, \mathrm{H}_{2} \mathrm{SO}_{4}$, etc.) are needed in the synthesis of porous carbon [22-28]. Zhang et al. have prepared 3D porous carbon through $\mathrm{KOH}$ activation using alkali lignin as raw material, which showed a capacitance of $165 \mathrm{~F} / \mathrm{g}$ at $0.05 \mathrm{~A} / \mathrm{g}$ [24]. Xu et al. synthesized interconnected hierarchical porous carbon from lignin-derived byproducts of bioethanol production by $\mathrm{H}_{2} \mathrm{SO}_{4}-\mathrm{KOH}$ joint hydrothermal carbonization, achieving a capacitance of $312 \mathrm{~F} / \mathrm{g}$ at $1.0 \mathrm{~A} / \mathrm{g}$ [27]. However, the overuse of highly corrosive reagents induces an excess of micropores that are inaccessible for electrolyte ions, and it is hard to recycle, which hinders the large-scale production. Even worse, the physical mixing of lignin and activator is easily to form large agglomerates between lignin and activator, a vital issue that often lowers the porosity of carbon product and limits the performance of supercapacitor. On the contrary, water-soluble lignosulfonate could be converted into porous carbon materials with good electrochemical properties by direct pyrolysis [29,30]. And lignosulfonate-derived carbons by conventional activation ( $\mathrm{KOH}, \mathrm{ZnCl}_{2}$, etc.) show improved electrochemical properties, of which the capacitances are higher than that of water-insoluble lignin-derived carbons [31,32]. In spite of the improved electrochemical properties, the aggregation and disorder of carbons are still inevitable. Thus, it is essential to develop a green and non-corrosive activation technique for the synthesis of advancing lignin-derived carbon.

Herein, we demonstrate a green and facile strategy for the synthesis of porous lignin-derived carbon (PLC) via zinc oxalate-assisted gas-exfoliation and in-situ templating for high-performance supercapacitors. Water-soluble sodium lignosulfonate is chosen as carbon precursor because its good water solubility and dispersion performance are beneficial for achieving well-dispersed with zinc oxalate. For another, the hydrophobic phenylpropane blocks and the hydrophilic sulfonate groups in amphiphilic lignosulfonate could induce self-assembly in solution to make it transform into lamellar structure. During in-situ hightemperature carbonization, the lamellar composite is gradually exfoliated by the constant gases $\left(\mathrm{CO}, \mathrm{CO}_{2}\right)$ released by zinc oxalate, which contributes to breaking the compact lignin skeleton effectively. Simultaneously, the generated zinc oxide nanoparticles act as template to produce numerous hierarchical pores. Surprisingly, the as-prepared samples exhibit 2D quasi-nanosheet architecture with a large surface area and rational pore distribution, which facilitates the diffusion and transfer of electrolyte ions and provides adequate active sites for charge accumulation [33]. Consequently, PLC shows a high capacitance performance of $320 \mathrm{~F} / \mathrm{g}$ at $1.0 \mathrm{~A} / \mathrm{g}$ and excellent long-term durability in a three-electrode system, which is much superior to most previously reported lignin-, biomass-derived carbons, and other conventional carbon material. Also, the symmetric supercapacitor fabricated with PLC shows superior rate performance and a high energy density of $9.75 \mathrm{~W} \mathrm{~h} / \mathrm{kg}$ at a high-power density of $6157.9 \mathrm{~W} / \mathrm{kg}$ in a two-electrode system. This work offers a green, economical, and scalable method to synthesize advanced nanotextured carbon materials for energy storage and conversion, and also develops a new path for the valorization of biomass.

\section{Experimental section}

\subsection{Chemicals}

Sodium lignosulfonate (LS) were purchased from Chempack Co., Ltd of Russia. Sodium oxalate $\left(\mathrm{Na}_{2} \mathrm{C}_{2} \mathrm{O}_{4}\right)$ and polytetrafluoroethylene (PTFE) were purchased from Aladdin. Zinc nitrate hexahydrate ( $\mathrm{Zn}$ $\left(\mathrm{NO}_{3}\right)_{2} \cdot 6 \mathrm{H}_{2} \mathrm{O}$ ) and polyvinyl alcohol (PVA) were purchased from Alfa Asear. Ethanol was obtained from Guangdong Guanghua Sci-Tech Co., Ltd of China. Hydrochloric acid $(\mathrm{HCl})$, potassium hydroxide $(\mathrm{KOH})$ and all the other reagents were purchased from Guangzhou Chemical Reagent Factory of China. All reagents were used without further purification.

\subsection{Synthesis of porous lignin-derived carbon quasi-nanosheets}

$3.72 \mathrm{~g} \mathrm{Zn}\left(\mathrm{NO}_{3}\right)_{2} \cdot 6 \mathrm{H}_{2} \mathrm{O}$ and a certain quality of LS were firstly dissolved in $20 \mathrm{ml}$ deionized water respectively, then mixed and kept stirring. $1.68 \mathrm{~g} \mathrm{Na}_{2} \mathrm{C}_{2} \mathrm{O}_{4}$ was next dissolved in $60 \mathrm{ml}$ deionized water and dropwise added into the mixed solution above to obtain highly dispersed suspensions. Under vigorous stirring, ethanol was slowly added to the suspensions until precipitates were generated in quantity. The precipitates were then collected by centrifugation, washed by ethanol and dried at $50{ }^{\circ} \mathrm{C}$ overnight to acquire lignosulfonate/zinc oxalate ( $\mathrm{LS} / \mathrm{ZnC}_{2} \mathrm{O}_{4}$ ) composites. Subsequently, the $\mathrm{LS} / \mathrm{ZnC}_{2} \mathrm{O}_{4}$ composites were carbonized in a horizontal tube furnace from room temperature to $650{ }^{\circ} \mathrm{C}$ at $5{ }^{\circ} \mathrm{C} / \mathrm{min}$ for two hours under a constant $\mathrm{N}_{2}$ flow. Then the products were soaked in $1 \mathrm{M} \mathrm{HCl}$ solution to remove $\mathrm{ZnO}$ and other impurities. The obtained carbons were neutralized with deionized water and dried at $80{ }^{\circ} \mathrm{C}$ for $12 \mathrm{~h}$ in a vacuum oven. The as-prepared samples were denoted as PLC-650-X, where $\mathrm{X}$ represents the mass ratio 


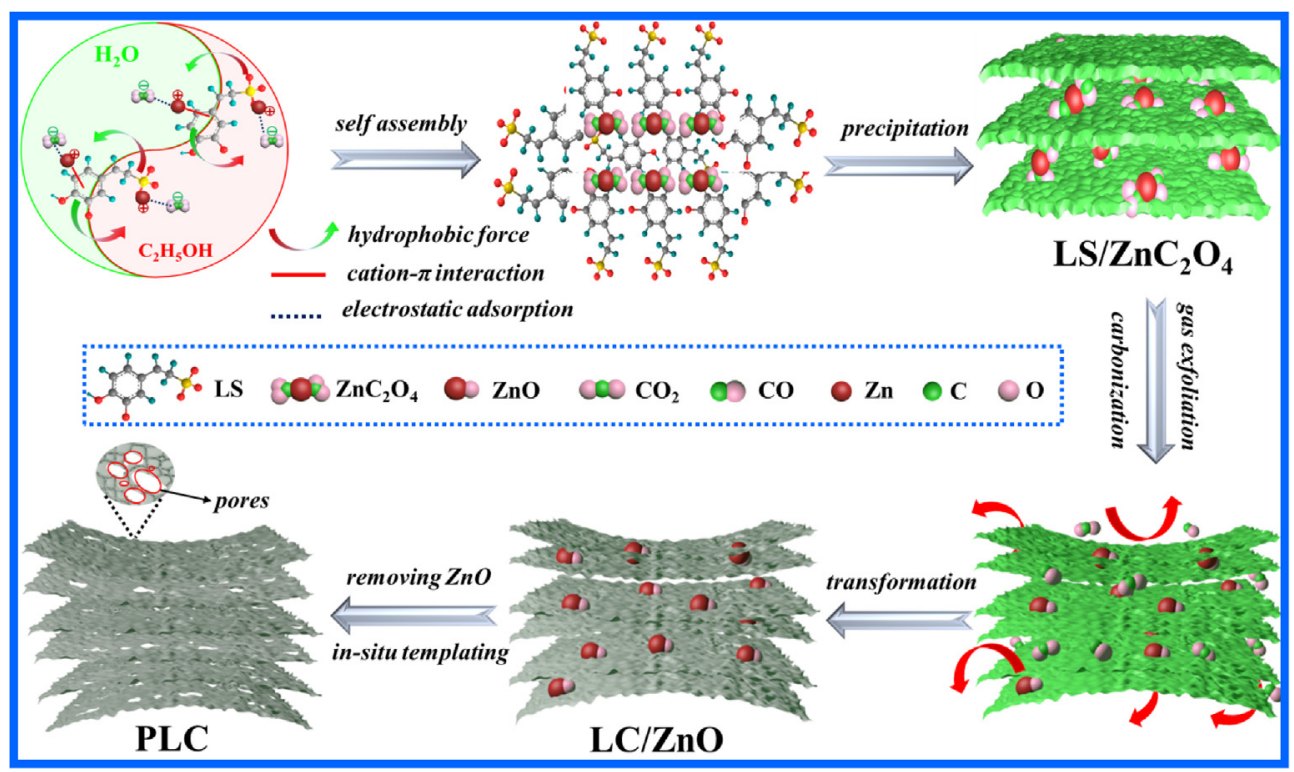

Scheme 1. Schematic illustration of the synthesis process for PLC.

of the generated $\mathrm{ZnC}_{2} \mathrm{O}_{4}$ to the LS used in the preparation $(\mathrm{X}=1,2$, and 3). For comparison, the sample prepared under $650{ }^{\circ} \mathrm{C}$ by directly pyrolyzing LS was named LC. To investigate the influence of the carbonization temperature, the samples PLC-550-2 and PLC-750-2 were also prepared as the carbonization temperature was set to 550 and $750{ }^{\circ} \mathrm{C}$ respectively.

\subsection{Material characterization}

The morphologies and microstructures of the samples were examined by scanning electron microscopy (SEM, SU8220, Hitachi) and transmission electron microscopy (TEM, JEM-2100F, JEOL, equipped with an energy dispersive spectroscopy). Thermogravimetric analysis (TGA) was performed on a thermal analyzer (STA449C, Netzsch) at a heating rate of $5{ }^{\circ} \mathrm{C} / \mathrm{min}$ under a $\mathrm{N}_{2}$ atmosphere to investigate the thermal decomposition behavior of $\mathrm{LS}, \mathrm{ZnC}_{2} \mathrm{O}_{4}$ and $\mathrm{LS} / \mathrm{ZnC}_{2} \mathrm{O}_{4}$ composites. X-ray diffraction (XRD) patterns of the samples were recorded by an X-ray power diffraction (D8 Advance, Bruker) with $\mathrm{Cu} \mathrm{Ka}$ wavelength of $0.1541 \mathrm{~nm}$. Raman spectra were recorded by a Raman spectrometer (LabR, AMA Ramis) with an excitation wavelength of $633 \mathrm{~nm}$. Brunauer-Emmett-Teller (BET) specific surface area of all samples was obtained by $\mathrm{N}_{2}$ adsorption-desorption at $77 \mathrm{~K}$ (TristarII3flex, Micrometrics). The specific micropore area was obtained by t-plot method, and the pore size distribution was collected by DFT model. X-ray photoelectron spectroscopy (XPS, Axis Ultra, Kratos Analytical Ltd.) measurements were performed to determine the surface elemental compositions of samples.

\subsection{Electrochemical measurements}

All the electrochemical measurements were performed on an electrochemical workstation (CHI660E, Chenhua, Shanghai). For working electrode preparation, $80 \mathrm{wt} \%$ active material (PLC), $10 \mathrm{wt} \%$ acetylene black and $10 \mathrm{wt} \%$ PTFE were mixed homogeneously in ethanol, then pressed onto nickel foam pieces with a pressure of $15 \mathrm{MPa}$ and dried at $60{ }^{\circ} \mathrm{C}$ in a vacuum oven overnight. The active mass loading is about $1.5 \mathrm{mg} / \mathrm{cm}^{2}$. A platinum foil and a saturated calomel electrode (SCE) were served as the counter and reference electrodes respectively in the three-electrode system. The cyclic voltammetry (CV), the galvanostatic charge/discharge (GCD) were conducted in $6 \mathrm{M} \mathrm{KOH}$ (voltage range from -1 to $0 \mathrm{~V}$ ). Then a symmetric supercapacitor was fabricated using the two identical electrodes with the active mass loading of $1.5 \mathrm{mg} / \mathrm{cm}^{2}$ and no-woven membrane separator (NKK-MPF30AC-100) for twoelectrode system tests. And the electrochemical behaviors of supercapacitor were studied in a voltage range from 0 to $1.2 \mathrm{~V}$ using PVA/ $\mathrm{KOH}$ gel as the electrolyte. The electrochemical impedance spectroscopy (EIS) was tested in a frequency range from $0.01 \mathrm{~Hz}$ to $100 \mathrm{kHz}$ with a voltage amplitude of $5 \mathrm{mV}$ under open circuit voltage.

The specific capacitance ( $C s, \mathrm{~F} / \mathrm{g}$ ) of electrodes based on the threeelectrode system and the two-electrode system was calculated from the charge/discharge curves by the following equations respectively:

$C s=I \Delta t / m \Delta V$

$C s=2 I \Delta t / m \Delta V$

where I (A) and $\Delta t(\mathrm{~s})$ are the charge/discharge current and discharge time, meanwhile $m(\mathrm{~g})$ is the mass of active materials of a single electrode, and $\Delta V(\mathrm{~V})$ is the voltage change within the discharge time $\Delta t$.

The specific energy density ( $E s, \mathrm{~W} \mathrm{~h} / \mathrm{kg}$ ) and specific power density (Ps, W/kg) based on the two-electrode system were calculated by the following formulas:

$E s=C s \Delta V^{2} /(8 \times 3.6)$

$P s=3600 E s / \Delta t$

where Cs is the specific capacitance calculated from the discharge curves based on two-electrode system, $\Delta V$ is the potential range, and $\Delta t$ is the discharge time.

\section{Results and discussion}

Scheme.1 shows the schematic diagram of the synthesis process for PLC. Firstly, LS, zinc ions, and oxalate ions disperse homogeneously in the water/ethanol solvent. On increasing the volume fraction of ethanol, the hydropholic benzene rings and hydrophilic sulfonate groups of LS gradually achieve orderly reverse under the induction of hydrophobic force. Meanwihle, cation- $\pi$ interactions predominately occur between LS and zinc ions, [34] and the zinc ions could combine with the oxalate ions through electrostatic adsorption. This promotes the self assembly between LS and the generated $\mathrm{ZnC}_{2} \mathrm{O}_{4}$. The further increase of ethanol results in a sharply decreased solubility for LS and enables the precipitation of the $2 \mathrm{D}$ lamellar-like $\mathrm{LS} / \mathrm{ZnC}_{2} \mathrm{O}_{4}$ composite. Finally, during carbonization in a $\mathrm{N}_{2}$ atmosphere, $\mathrm{ZnC}_{2} \mathrm{O}_{4}$ releases gases $\left(\mathrm{CO}, \mathrm{CO}_{2}\right)$ continuously to further exfoliate and cut thin the lignin, thus the $\mathrm{LS} / \mathrm{ZnC}_{2} \mathrm{O}_{4}$ composite can be easily transformed into the carbon/ 

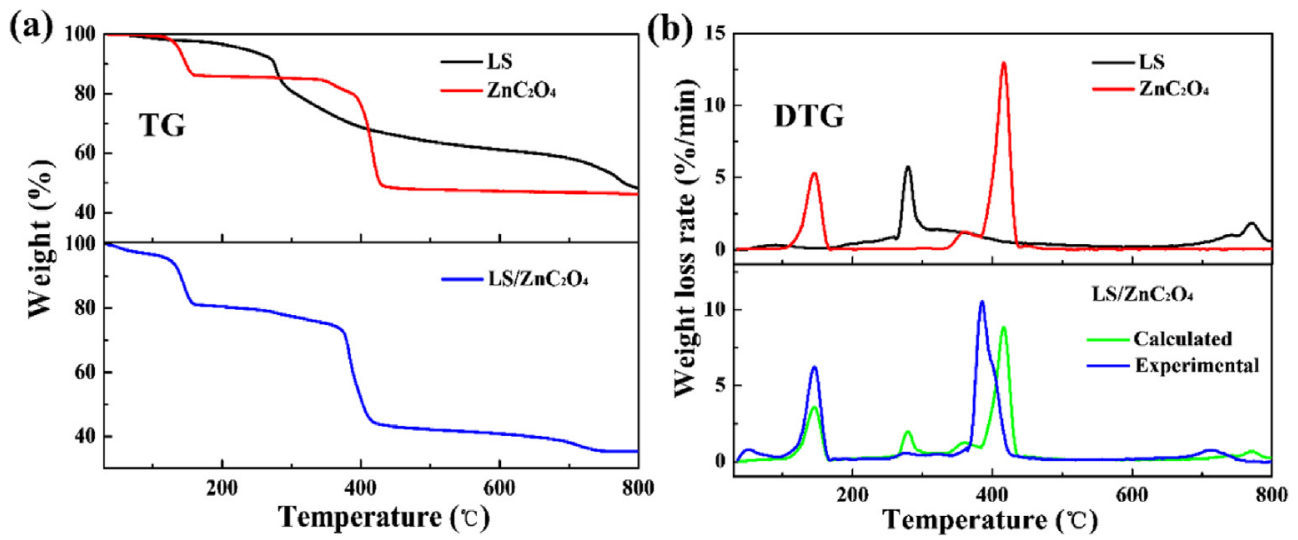

Fig. 1. TG (a) and DTG (b) analysis of $\mathrm{LS}, \mathrm{ZnC}_{2} \mathrm{O}_{4}$, and $\mathrm{LS} / \mathrm{ZnC}_{2} \mathrm{O}_{4}-2$.

$\mathrm{ZnO}$ hybrid. The nanosized $\mathrm{ZnO}$ templates can be also easily removed by acid treatment, leading to the formation of PLC with 2D porous nanosheet structure.

In order to study the reciprocity between the lignin and the activator during carbonization, TGA was applied to figure out the pyrolysis behavior of $\mathrm{LS}, \mathrm{ZnC}_{2} \mathrm{O}_{4}$, and $\mathrm{LS} / \mathrm{ZnC}_{2} \mathrm{O}_{4}$ composite. The weight loss of LS from $25^{\circ} \mathrm{C}$ to $800{ }^{\circ} \mathrm{C}$ is mainly divided into three stages, as shown in Fig. 1a. The first weight loss (ca. 7\%) from room temperature to $200{ }^{\circ} \mathrm{C}$ is attributed to the elimination of moisture and the volatile matters. The second weight loss (ca. $28 \%$ ) in the range of $200-600^{\circ} \mathrm{C}$ shows a sharp weight loss rate peak at $280{ }^{\circ} \mathrm{C}$ (Fig. 1b). In this stage, most unstable oxygen-containing functional groups, together with some small sulfurcontaining molecules decomposed to form an amorphous carbon skeleton. A weight loss (ca. 15\%) in the range of $600-800{ }^{\circ} \mathrm{C}$ with the maximum weight loss rate at $770{ }^{\circ} \mathrm{C}$ (Fig. 1b) occurred in the third stage, where the remained oxygen-containing groups continued to decompose, and the carbon skeleton could react with the released $\mathrm{CO}$ as well as $\mathrm{CO}_{2}$ [29]. For $\mathrm{ZnC}_{2} \mathrm{O}_{4}$, a weight loss ends at $150{ }^{\circ} \mathrm{C}$, and a weight loss in the range of $350-420^{\circ} \mathrm{C}$ are observed. The former corresponds to the elimination of combined water and the latter results from the decomposition of $\mathrm{ZnC}_{2} \mathrm{O}_{4}$. Dramatically, the latter well matches with the second weight loss of LS, which stimulates the co-pyrolysis between LS and $\mathrm{ZnC}_{2} \mathrm{O}_{4}$.

Fig. 1b shows the experimental and calculated weight loss rates for $\mathrm{LS} / \mathrm{ZnC}_{2} \mathrm{O}_{4}$ composite, in which the calculated rate was obtained by assuming that the weight loss for $\mathrm{LS} / \mathrm{ZnC}_{2} \mathrm{O}_{4}$ composite was a summation of the values for $\mathrm{LS}$ and $\mathrm{ZnC}_{2} \mathrm{O}_{4}$. Especially, the experimental rate does not display a sharp peak at $280{ }^{\circ} \mathrm{C}$, confirming strong interactions were formed between $\mathrm{LS}$ and $\mathrm{ZnC}_{2} \mathrm{O}_{4}$ during the self-assembly owing to the anion- $\pi$ interaction and electrostatic adsorption, thus leading to a delayed decomposition for LS. Moreover, the peaks around $420{ }^{\circ} \mathrm{C}$ and $770{ }^{\circ} \mathrm{C}$ of calculated curve shift to lower temperature in the experimental curve, and the peak around $380{ }^{\circ} \mathrm{C}$ in the experimental curve is higher than that of the calculated curve, implying a mutual promotion in pyrolysis.

The evolution of micromorphologies during the preparation was characterized by scanning electron microscope (SEM). As shown in Fig. S1a \& b, both $\mathrm{ZnC}_{2} \mathrm{O}_{4}$ and LS powder are irregular bulk structure and show terrible dispersibility. Self-assembling in the water/ethanol solution, the obtained $\mathrm{LS} / \mathrm{ZnC}_{2} \mathrm{O}_{4}$ composites show ellipsoidal sheetstacking structure with a diameter of 4-5 $\mu \mathrm{m}$ (Fig. 2a, Fig. S1c \& e). The $\mathrm{XRD}$ pattern of the composite in Fig. S2 indicates the existence of $\mathrm{ZnC}_{2} \mathrm{O}_{4} \cdot 2 \mathrm{H}_{2} \mathrm{O}$, but some diffraction peaks were covered by LS. After carbonizing at $650{ }^{\circ} \mathrm{C}$, well-defined characteristic diffraction peaks of $\mathrm{ZnO}$ appeared in Fig. S2, indicating the composites were successfully transformed into carbon/ZnO hybrids (Fig. 2b, Fig. S1d \& f). The generated zinc oxide nanoparticles were embedded in the carbon layers to prevent from collapse and gathering.
Interestingly, PLC-650-2 exhibits 2D interconnected quasi-nanosheet structure (Fig. 2c) with numerous macropores after removing $\mathrm{ZnO}$, while pure LC prepared by direct pyrolysis of lignosulfonate shows a quasi-sphere shape with few pores (Fig. S3a1). And SEM images of other PLC-650 (Fig. S3b1 \& c1) exhibit the similar structure as PLC-6502. Transmission electron microscope (TEM) images (Fig. 2d, Fig. S3b2 \& c2) further demonstrate the existence of abundant micropores and mesopores inside PLCs, indicating the porosity was significantly enhanced with the assistant of $\mathrm{ZnC}_{2} \mathrm{O}_{4}$. These above results confirm that PLCs have a hierarchical porous structure composed of macropores, mesopores, and micropores. And this 2D porous nanosheet structure could not only provide efficient active sites for charge accumulation but also shorten the paths for electrolyte diffusion [33]. The high-resolution TEM (HRTEM) images (Fig. 2e, Fig. S3b3 \& c3) further show that PLCs possess obvious graphitized layers with a lattice space of about 0.357 , 0.348 , and $0.351 \mathrm{~nm}$, respectively. Fig. S4 shows the digital photos of PLC-650-2 and other commercial carbon materials, indicating that the ratio of surface area to weight of PLC-650-2 is comparable with commercial multilayer graphene. Fig. $1 \mathrm{f}$ displays that $\mathrm{C}$, $\mathrm{O}$, and $\mathrm{S}$ are distributed evenly in PLC-650-2. Overall, it can be inferred reasonably that the quasi-nanosheet structure results from the exfoliation of gaseous products ( $\mathrm{CO}, \mathrm{CO}_{2}, \mathrm{H}_{2} \mathrm{O}$, etc.) released by the decomposition of $\mathrm{ZnC}_{2} \mathrm{O}_{4}$, [35] and the abundant nanosized pores derive from the activation of gas and the $\mathrm{ZnO}$ template.

On the other hand, given that the carbonization temperature is an important parameter for the microstructure of biomass carbonized products, $[29,30]$ we further explore the correlation between the carbonization temperature and the microstructure of the resulting carbons in this work. As shown in Fig. S5, PLC-550-2 shows a 2D nanosheet structure but with a low porosity due to inadequate pyrolysis of carbon precursor. When the temperature was elevated to $750{ }^{\circ} \mathrm{C}$, the resulting carbon shows a 3D sponge-like porous structure. The pores are widened because the generated $\mathrm{ZnO}$ nanoparticles tend to migrate and sinter at high temperature.

The textural properties of the prepared samples were further measured by $\mathrm{N}_{2}$ adsorption-desorption at $77 \mathrm{~K}$. Fig. 3a shows that all the lignin-derived carbons except LC reveal a typical type-IV isotherm with a distinct type-H3 hysteresis loop at the relative high pressure (P/ $\mathrm{P}_{0}>0.6$ ), indicating the co-existence of macropores and mesopores. Meanwhile, PLCs exhibit a sharp adsorption knee at the low relative pressure $\left(\mathrm{P} / \mathrm{P}_{0}<0.01\right)$, which suggests the existence of large quantities of micropores. Moreover, the DFT pore size distribution curves in Fig. 3b reveal that PLCs possess a hierarchical porous structure with a micropore distribution $(0.5-2 \mathrm{~nm})$ and a broad mesopore distribution $(2-20 \mathrm{~nm})$, which is consistent with the result of TEM.

In more detail, the textural properties of LC and PLCs are listed in Table 1. PLC-650-2 shows a large SSA of $1069 \mathrm{~m}^{2} / \mathrm{g}$ and a high total pore volume of $1.375 \mathrm{~cm}^{3} / \mathrm{g}$, together with reasonable micropore and 

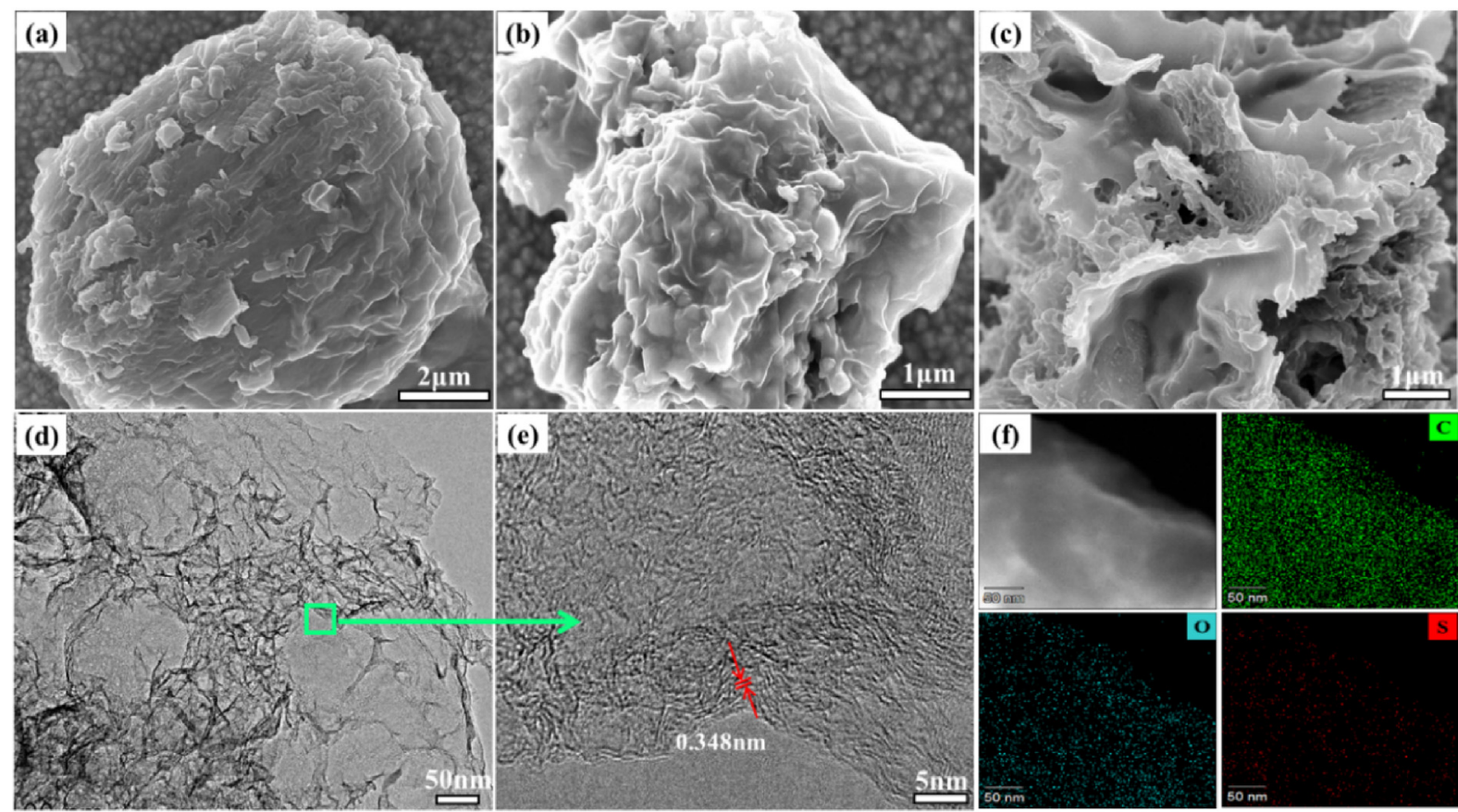

Fig. 2. SEM images: $\mathrm{LS} \mathrm{ZnC}_{2} \mathrm{O}_{4}-2$ (a), LC/ZnO-2 (b), PLC-650-2 (c); TEM image (d), HRTEM image (e), and corresponding elemental mapping image (f) of C, O, and S of PLC-650-2.

mesopore surface area, suggesting the textural property could be optimized by regulating the mass ratio of raw material. Notably, its mesopore volume reaches $1.198 \mathrm{~cm}^{3} / \mathrm{g}$, taking up $87.1 \%$ of total pore volume, very possibly because the $\mathrm{ZnO}$ nanoparticles act as template favoring to the formation of mesopores. This typical mesopore-dominated porous carbon is different from the microporous carbon materials through $\mathrm{KOH}$ activation $[23,24,27,28]$. Large quantities of mesopores are favorable for ion buffer, thus facilitating the diffusion and transport of electrolyte. [36] More importantly, higher SSA and larger pore volume are achieved by $\mathrm{ZnC}_{2} \mathrm{O}_{4}$-assisted exfoliation and templating comparing with direct pyrolysis. These above results demonstrate that the gas-exfoliation and in-situ templating of $\mathrm{ZnC}_{2} \mathrm{O}_{4}$ are essential for the formation of the hierarchical porous quasi-nanosheet. The high SSA and large pore volume are beneficial in enhancing electrochemical performances of carbon electrode materials.

Fig. S6a \& b show the nitrogen adsorption-desorption isotherms and DFT pore size distribution of the samples prepared under different temperature. The SSA and the total pore volume are increased with the

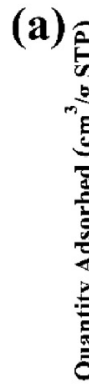

(c)
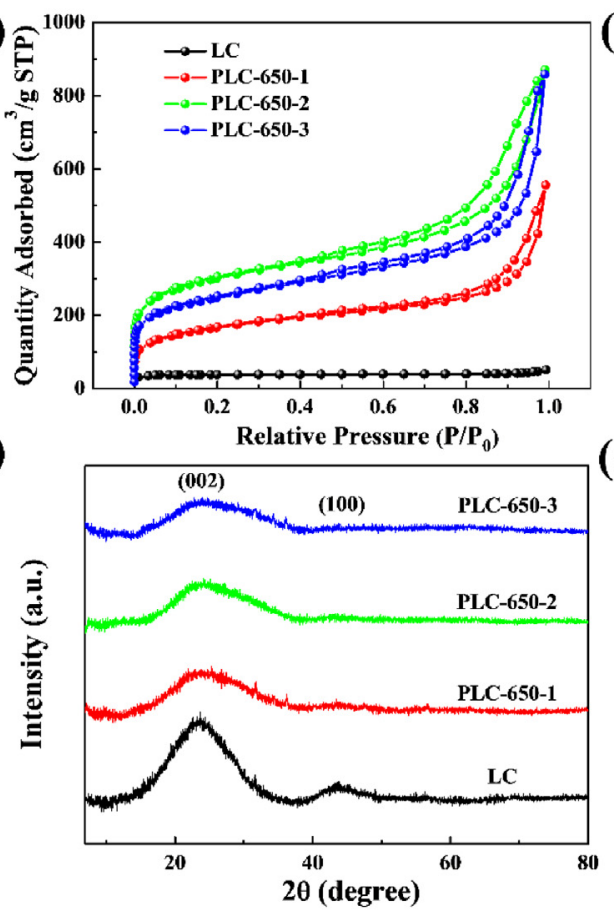

(b)

b)

(d)
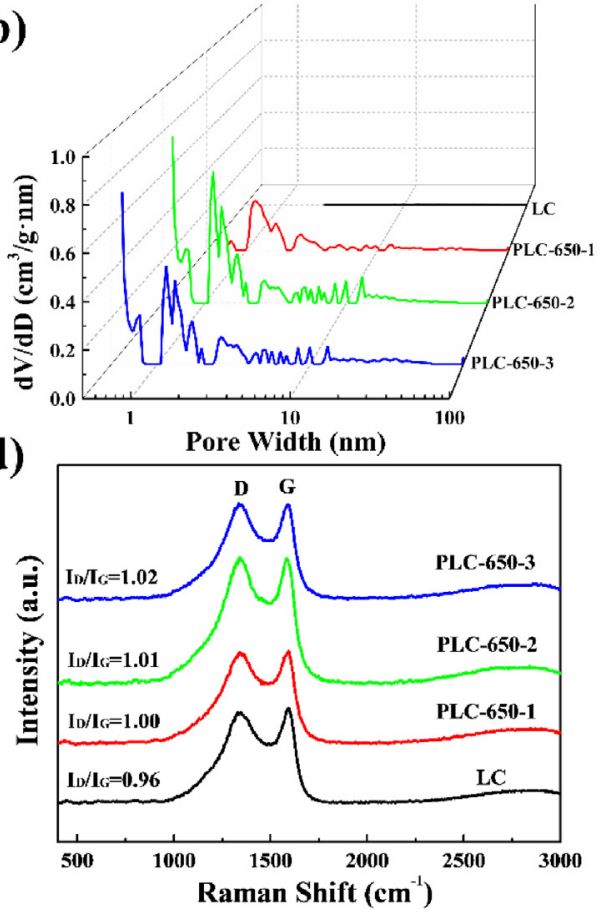

Fig. 3. (a) Nitrogen adsorption-desorption isotherms, (b) DFT pore size distribution, (c) XRD patterns, and (d) Raman spectra of LC, PLC-650-1, PLC-650-2, and PLC650-3. 
Table 1

Textural properties of LC and PLC. ${ }^{a}$

\begin{tabular}{lllllllll}
\hline Samples & \multicolumn{2}{l}{ SSA $\left(\mathrm{m}^{2} / \mathrm{g}\right)$} & \multicolumn{4}{c}{ Pore Volume $\left(\mathrm{cm}^{3} / \mathrm{g}\right)$} & \multicolumn{2}{l}{ Percentage (\%) } \\
\cline { 2 - 9 } & $\mathrm{S}_{\text {BET }}$ & $\mathrm{S}_{\text {micro }}$ & $\mathrm{S}_{\text {meso }}$ & $\mathrm{V}_{\text {total }}$ & $\mathrm{V}_{\text {micro }}$ & $\mathrm{V}_{\text {meso }}$ & $\begin{array}{l}\mathrm{V}_{\text {micro }} / \\
\mathrm{V}_{\text {total }}\end{array}$ & $\begin{array}{l}\mathrm{V}_{\text {meso }} / \\
\mathrm{V}_{\text {total }}\end{array}$ \\
\hline LC & 75 & 63 & 12 & 0.046 & 0.023 & 0.023 & 50.0 & 50.0 \\
PLC-650-1 & 585 & 128 & 457 & 0.867 & 0.060 & 0.807 & 6.9 & 93.1 \\
PLC-650-2 & 1069 & 406 & 663 & 1.375 & 0.177 & 1.198 & 12.9 & 87.1 \\
PLC-650-3 & 872 & 265 & 607 & 1.134 & 0.120 & 1.014 & 10.6 & 89.4 \\
PLC-550-2 & 232 & 35 & 197 & 0.311 & 0.005 & 0.306 & 1.6 & 98.4 \\
PLC-750-2 & 1300 & 140 & 1160 & 2.031 & 0.070 & 1.961 & 3.4 & 96.6 \\
\hline
\end{tabular}

$\mathrm{V}_{\text {total: }}$ total volume; $\mathrm{V}_{\text {micro }}$ : micropore volume; $\mathrm{V}_{\text {meso }}$ : mesopore volume.

${ }^{\text {a }} \mathrm{S}_{\mathrm{BET}}$ : total BET specific surface area; $\mathrm{S}_{\text {micro }}$ : micropore surface area; $\mathrm{S}_{\text {meso }}$ : mesopore surface area.

increased carbonization temperature, but the micropore surface area and volume show an evident decrement as the carbonization temperature elevates to $750{ }^{\circ} \mathrm{C}$. As shown in Fig. S6b, the pore distribution of PLC-750-2 in the range of 0.5-2 nm sharply decreases, indicating that the micropores are reduced during the pore size expanding process, which is in good agreement with the result of SEM. Although PLC-750-2 shows the highest SSA of $1300 \mathrm{~m}^{2} / \mathrm{g}$ and the maximum total pore volume of $2.031 \mathrm{~cm}^{3} / \mathrm{g}$, the limited micropore distribution suppresses the improvement of electrochemical properties. [37]

XRD and Raman spectroscopy were used to investigate the structural characteristics of the prepared samples. As shown in Fig. 3c, broad $\left(\begin{array}{lll}0 & 0 & 2\end{array}\right)$ and weak ( $\left.\begin{array}{lll}1 & 0 & 0\end{array}\right)$ diffraction peaks are located at ca. $23^{\circ}$ and ca. $44^{\circ}$, indicating the amorphous carbon structure of samples. In comparison to LC $\left(\begin{array}{lll}0 & 0 & 2\end{array}\right)$, the peak intensity for PLCs is weakened, and the starting points shift to the larger angle, suggesting an expanding carbon interlayer distance duo to the gas-exfoliation. As depicted in Fig. 3d, two distinct peaks can be observed in the Raman spectra at about $1350 \mathrm{~cm}^{-1}$ and $1590 \mathrm{~cm}^{-1}$, indexed to the $\mathrm{D}$ band and the $\mathrm{G}$ band. The $\mathrm{D}$ band represents disordered carbon, and the $\mathrm{G}$ band is related to the ordered graphite carbon structure [38]. The intensity ratio of $\mathrm{D}$ band to $\mathrm{G}$ band $\left(\mathrm{I}_{\mathrm{D}} / \mathrm{I}_{\mathrm{G}}\right)$ describes the degree of graphitization of carbon materials. It can be found that the values of $\mathrm{I}_{\mathrm{D}} / \mathrm{I}_{\mathrm{G}}$ for PLCs are almost the same, implying the effect of the dosage of LS on graphitization degree could be neglected. Nevertheless, the ratios of $\mathrm{I}_{\mathrm{D}} / \mathrm{I}_{\mathrm{G}}$ show an abnormal increase with the increased carbonization temperature (Fig. S6c), indicating lower heat treatment temperature is in favor of a more graphitic structure, very possibly because more defects were introduced by the etching of $\mathrm{ZnO}$ in high temperature.

$\mathrm{X}$-ray photoelectron spectroscopy (XPS) was used to determine the surface elemental compositions of the as-prepared carbons. The overall XPS survey spectra in Fig. 4a and S6d show the co-existence of the strong C 1s peak and O 1s peak, and trace of sulfur is also observed. Normalized quantitative results of the surface elemental contents are listed in Table S1. At the same carbonization temperature, PLCs show higher carbon contents (96.30-96.79 at\%) than LC (95.09 at\%), indicating more oxygen-containing functional groups were removed with the help of $\mathrm{ZnC}_{2} \mathrm{O}_{4}$. And the carbon content increases to $97.44 \mathrm{at} \%$ from 92.99 at $\%$ and the oxygen content decreases from 6.68 at $\%$ to 2.35 at $\%$ when the carbonization temperature increases from $550{ }^{\circ} \mathrm{C}$ to $750{ }^{\circ} \mathrm{C}$, implying the unstable oxygen-containing groups are more eliminated at higher temperature. As shown in Fig. 4b, the high-resolution C 1s spectra of PLC-650-2 can be divided into five peaks, which are assigned to $\mathrm{sp}^{2}$-bonded carbon (284.6 eV), $\mathrm{sp}^{3}$-bonded carbon (285.6 eV), carbon in alcohol groups (286.5 eV), carbon in carbonyl groups (287.2 eV), and carbon in carboxyl $(288.8 \mathrm{eV})$, respectively $[39,40]$. The high-resolution $\mathrm{O}$ 1s spectra in Fig. $5 \mathrm{c}$ further confirms the presence of $\mathrm{C}-\mathrm{OH} /$ $\mathrm{C}-\mathrm{O}-\mathrm{C}, \mathrm{C}=\mathrm{O}$, and $-\mathrm{COOH}$ groups, located at $531.0 \mathrm{eV}, 532.3 \mathrm{eV}$, and $533.6 \mathrm{eV}$. Similarly, the S 2 p spectra can be split into three peaks at $163.9 \mathrm{eV}, 165.0 \mathrm{eV}$, and $169.1 \mathrm{eV}$, which represents the spin-orbital of $\mathrm{S}$ $2 \mathrm{p}_{3 / 2}, \mathrm{~S} 2 \mathrm{p}_{1 / 2}$ (C-S-C), and oxidized S (C-SO $\left.\mathrm{X}^{-} \mathrm{C}\right)$, respectively $[41,42]$. It is reported that oxygen- and sulfur-containing functional groups not only enhance the affinity between the surface of carbon and electrolyte but also introduce additional pseudocapacitance via redox reactions $[43,44]$.

The capacitive performances of all samples were estimated by using a three-electrode system in $6 \mathrm{M} \mathrm{KOH}$. Fig. 5a and S6a display the cyclic voltammetry (CV) curves of all samples at the scan rate of $5 \mathrm{mV} / \mathrm{s}$. All the CV curves take an approximately rectangular shape, revealing the predominance of double-layer capacitance. Noteworthily, a weakly broad hump between $-0.7 \mathrm{~V} \sim-0.5 \mathrm{~V}$ occurs in the CV curve of PLC$650-2$ caused by the redox reactions of heteroatomic functional groups [45]. By comparison, PLC-650-2 has the largest encircled area and possesses the best capacitance characteristic among the samples. The galvanostatic charge-discharge (GCD) curves at the current density of $1.0 \mathrm{~A} / \mathrm{g}$ for all samples in Fig. 5b and S7b display symmetrical triangular shapes, suggesting good electrochemical reversibility. Calculated based on the Eq. (1), the specific capacitances are 156, 209, 320, 240, 193 and 244 F/g for LC, PLC-650-1, PLC-650-2, PLC-650-3, PLC-550-2 and PLC-750-2, respectively, of which PLC-650-2 shows the highest value. Its capacitance is comparable with previously reported carbons derived from lignin or other biomass (Table S2) and even higher than that of graphene-based materials (Table S3). It is noteworthy that PLC750-2 exhibits the highest SSA but shows a moderate specific capacitance, and the reason lies in the deceleration for the diffusion of electrolyte caused by the limited micropore area and volume.

Fig. 5c shows the CV curves of PLC-650-2 tested at various scan rates, and all the curves remain a symmetric and rectangular shape even at a high scan rate of $200 \mathrm{mV} / \mathrm{s}$, implying its good rate performance. Figs. S7 and S8 show the CV curves of other samples, LC and PLC-550-2 show more bending deformation at high scan rate owing to their poor porosity. Also, GCD curves of all samples presented in Fig. 5d, Figs. S7, and S8 display typical capacitor triangle shapes with a small IR drop at high current density. PLCs show higher discharge time, indicating they have higher specific capacitance than LC. Their superior electrochemical properties are ascribed to the enhanced SSA and the abundant pore structures inherited from the refined self-assembly process.

Fig. 5e and S9 plot the specific capacitance of all samples calculated from GCD curves at various current densities. It is an inherent phenomenon that the specific capacitance declines with the increased current density because the electrolyte ions do not have enough time to diffuse to the surface of the electrode in the ultrafast charging/discharging process. The specific capacitance of PLC-650-2 achieves $365 \mathrm{~F} / \mathrm{g}$ at $0.5 \mathrm{~A} / \mathrm{g}$, and still supplies a satisfactory specific capacitance of $260 \mathrm{~F} / \mathrm{g}$ at $20.0 \mathrm{~A} / \mathrm{g}$ (71.2\% capacitance retention), indicating an excellent rate capacity triggered by the well-developed mesopores. But the specific capacitance of LC decreases sharply and maintains only 60 $\mathrm{F} / \mathrm{g}$ at $20.0 \mathrm{~A} / \mathrm{g}$, resulting in a rate capability of $35.9 \%$. Moreover, Fig. 5 f reveals that PLC-650-2 has good cycling durability with $93.5 \%$ capacitance retention after 10,000 cycles at $5.0 \mathrm{~A} / \mathrm{g}$ and its coulombic efficiency almost reaches $100 \%$.

Based on the discussion above, the mechanism of superior electrochemical performances of PLC-650-2 is illustrated in Fig. $5 \mathrm{~g}$ with highlighted ion diffusion and electron transfer pathways. Firstly, PLC650-2 shows a porous quasi-2D nanosheet architecture with abundant active surface for charge accumulation [46]. And this open structure shortens the pathway for electrolytes diffusion, where $\mathrm{K}^{+}$and $\mathrm{OH}^{-}$ ions could not only transport through the interlayer but also shuttle in the vertical direction through the pores [47]. Secondly, the large mesopore volume significantly accelerates the ion transport kinetics by buffering many electrolytes, leading to low ion transport resistance [48]. In particular, the intact sheets also provide unimpeded pathways for electrons transfer, endowing PLC-650-2 with high conductivity. Thirdly, the interconnected 2D sheet structure shows good mechanical properties so that its structure is not easy to destroy during long-term cyclic charging and discharging. All in all, these factors synergistically 

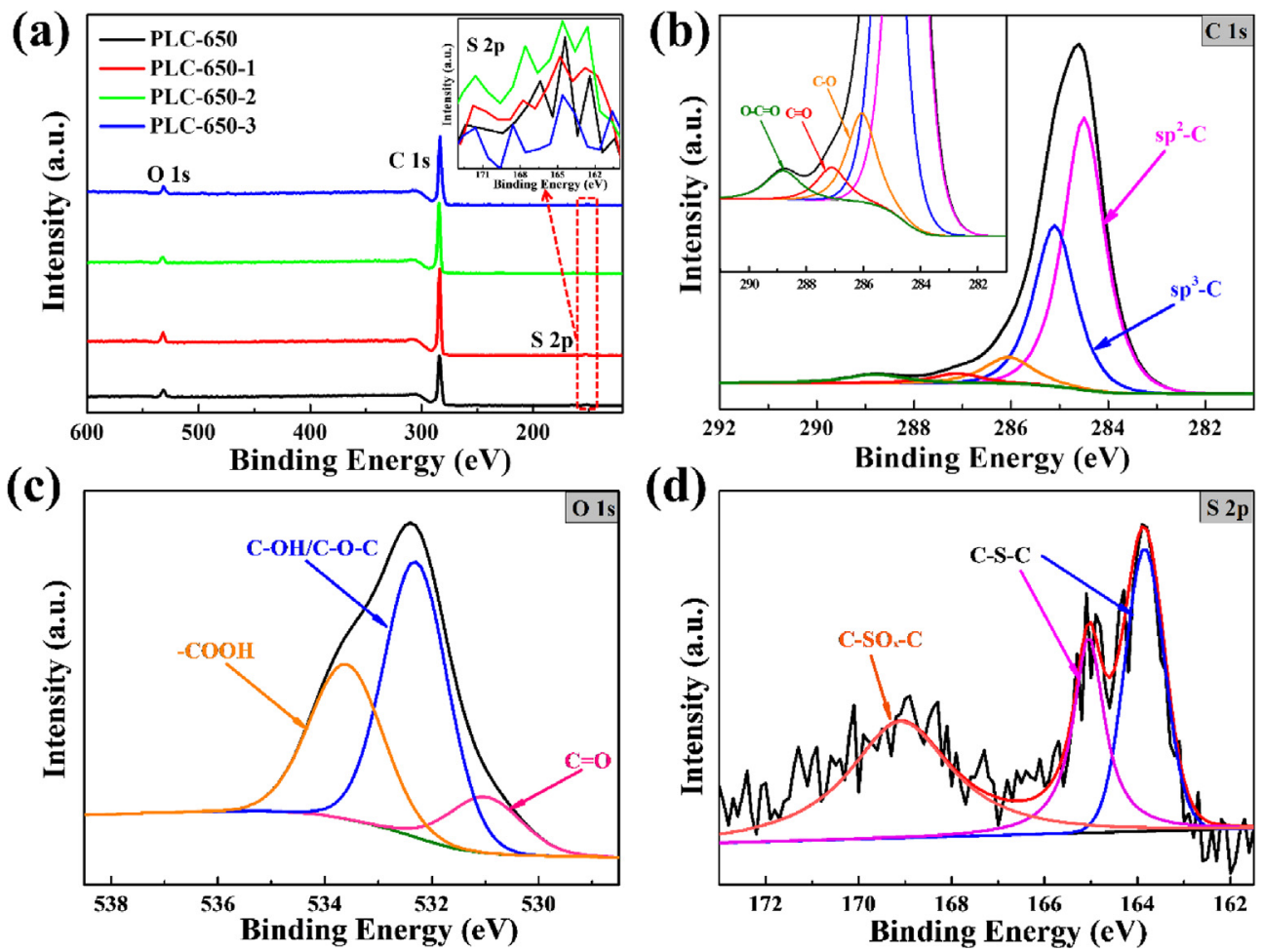

Fig. 4. (a) XPS survey spectra of LC, PLC-650-1, PLC-650-2, and PLC-650-3, (b) high-resolution C 1s, (c) O 1s, and (d) S 2p XPS spectra of PLC-650-2.

result in greatly enhanced electrochemical performances for PLC-650-2, including high specific capacitance, good rate capability, and superior cycling durability.

To investigate the practical application of PLC for supercapacitors, a symmetric supercapacitor was assembled with two identical PLC-650-2 electrodes using PVA/KOH gel as the electrolyte. Fig. 6a shows the CV curves tested in a voltage range of $0-1.2 \mathrm{~V}$, and the well-maintained quasi-rectangular shapes indicate an ideal electrical double-layer capacitor performance with superior rate capability. The GCD curves at various current densities are presented in Fig. 6b, and all the curves are nearly linear with a negligible IR drops, suggesting its low ESR for high power delivery. The specific capacitance of PLC-650-2 at various current densities can be calculated based on the Eq. (2), of which the values are $274 \mathrm{~F} / \mathrm{g}, 264 \mathrm{~F} / \mathrm{g}, 249 \mathrm{~F} / \mathrm{g}, 225 \mathrm{~F} / \mathrm{g}, 218 \mathrm{~F} / \mathrm{g}$, and $200 \mathrm{~F} / \mathrm{g}$, assigned to the current density of $0.5 \mathrm{~A} / \mathrm{g}, 1.0 \mathrm{~A} / \mathrm{g}, 2.0 \mathrm{~A} / \mathrm{g}, 5.0 \mathrm{~A} / \mathrm{g}$, $10.0 \mathrm{~A} / \mathrm{g}$, and $20.0 \mathrm{~A} / \mathrm{g}$. The superior rate capability with capacitance retention of $73.0 \%$ is attributed to the low diffusion resistance and short transportation distance for electrolyte ions [49].

Electrochemical impedance spectroscopy for symmetric supercapacitors was carried out to further study the frequency response characteristics of samples. Fig. $6 \mathrm{c}$ displays the Nyquist plots of all the symmetric supercapacitors, and the relevant fitting equivalent circuit is inserted in Fig. 6c. All the plots are divided into three portions, including a semicircle in the high frequency region, a Warburg curve with an angle close to $45^{\circ}$ at the medium frequency region, and a line parallel to the virtual axis in the low frequency region. Table S4 summarizes the charge transfer resistance (Rct), Warburg resistance (Zw) and equivalent series resistance (ESR) of all supercapacitors according to the equivalent circuit model fitting. The diameter of the semicircle represents the Rct between the electrode and electrolyte interface,[50] and the fitting Rct for PLC-650-2 based supercapacitor is $0.266 \Omega$, which is lower than $3.220 \Omega$ (LC), $0.808 \Omega$ (PLC-650-1), and $0.568 \Omega$ (PLC-650-3). The lowest Rct of PLC-650-2 based supercapacitor results from two factors: (1) the abundant micro and mesopores create fast diffusion path for the electrolyte transportation; (2) the quasi-nanosheet structure shortens the length of electrolyte diffusion, permitting the transport of ions between horizontal and vertical carbon layers [51]. Additionally, PLC-650-2 based supercapacitor also exhibits the smallest $\mathrm{Zw}$ of $0.131 \Omega$, indicating its high porosity and thin thickness of the electrode. The steep linear curves of PLC-based supercapacitors in the low frequency region manifest an ideal capacitive performance with low diffusion resistance. The intersection of the semicircle and the real axis in the high frequency region stands for ESR, consisting of the resistance of active materials, the contact resistance between active materials and the current collector, and the resistance of electrolyte. All the PLC-based devices have a smaller ESR (0.542 $0.769 \Omega$ ) than LC-based device, indicating their good electrical conductivity and great potential in supercapacitor application.

Ragone plot of PLC-650-2 based symmetric supercapacitor is illustrated in Fig. $6 \mathrm{~d}$. The supercapacitor has slight decay of energy density with increased power density, and it offers a high energy density of $13.7 \mathrm{~W} \mathrm{~h} / \mathrm{kg}$ at a power density of $167.2 \mathrm{~W} / \mathrm{kg}$. The energy density keeps $9.75 \mathrm{~W} \mathrm{~h} / \mathrm{kg}$ at an ultrahigh power density of $6157.9 \mathrm{~W} / \mathrm{kg}$. These results indicate the PLC-650-2 based supercapacitor has outstanding energy-power characteristic even better than the reported lignin- or biomass-derived carbon-based supercapacitors [22,24,29,30,52-58].

\section{Conclusions}

In summary, a porous carbon quasi-nanosheet has been successfully synthesized from lignosulfonate by a green and facile gas-exfoliation and in-situ templating method. Compared with the traditional chemical activation, this technique innovatively adopts non-toxic and low-corrosive zinc oxalate as pore-creating substance, which avoids the damage of equipment and the shrink of lignin during carbonization. The as-obtained PLC-650-2 has a large specific surface area, rich porosity, and rational pore size distribution, which not only offers channels for quick diffusion of electrolyte but also provides adequate active sites for charge storage. Therefore, PLC-650-2 exhibits an outstanding specific capacitance of $320 \mathrm{~F} / \mathrm{g}$ at $1.0 \mathrm{~A} / \mathrm{g}$ in a three-electrode system. Also, the assembled PLC-650-2-based symmetric supercapacitor shows a superior 

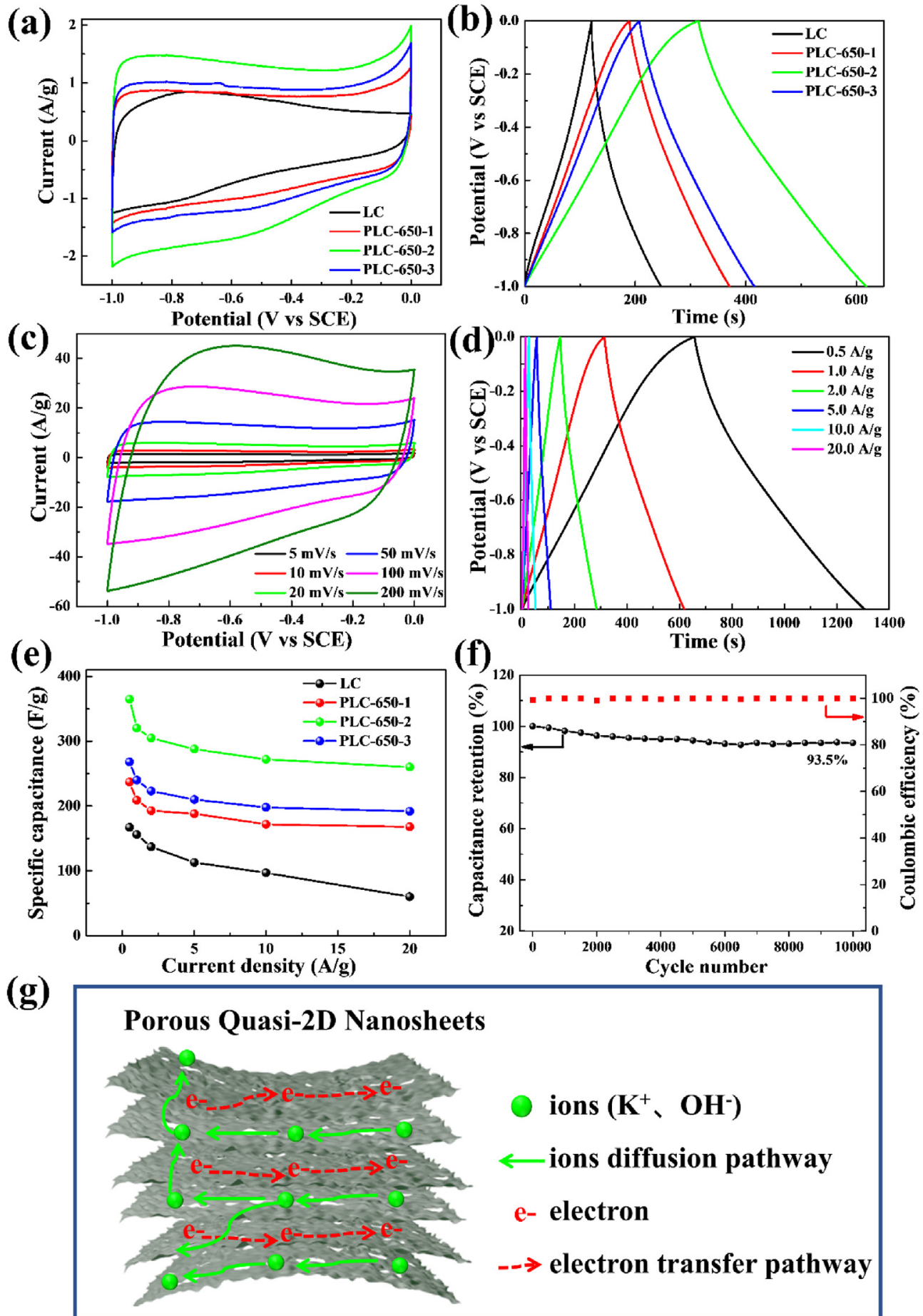

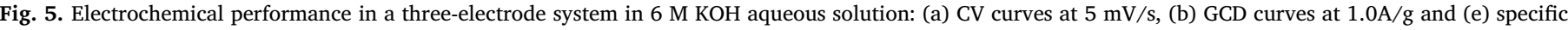

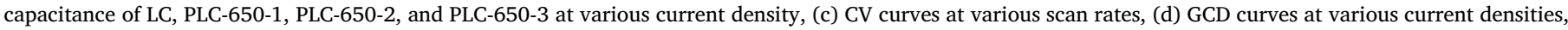

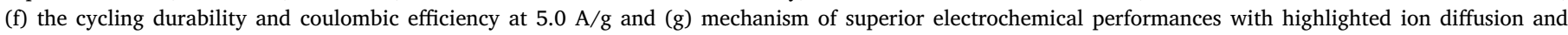
electron transfer pathways of PLC-650-2.

energy density of $9.75 \mathrm{~W} \mathrm{~h} / \mathrm{kg}$ at a high-power density of $6157.9 \mathrm{~W} / \mathrm{kg}$ in a two-electrode system. This work proposes a new way to convert industrial lignin into nanoporous carbon materials for energy storage.

\section{Declaration of Competing Interest}

The authors declare that they have no known competing financial interests or personal relationships that could have appeared to influence the work reported in this paper.

\section{Acknowledgements}

The authors would like to acknowledge the National Key Research and Development Program of China (2018YFB1501503), the National Natural Science Foundation of China (NSFC) (No. 21878114, 21690083, 21908071), and Natural Science Foundation of Guangdong Province (2018B030311052, 2017B090903003).

Fu F. B. and Yang D. J. have contributed equally to this work. 

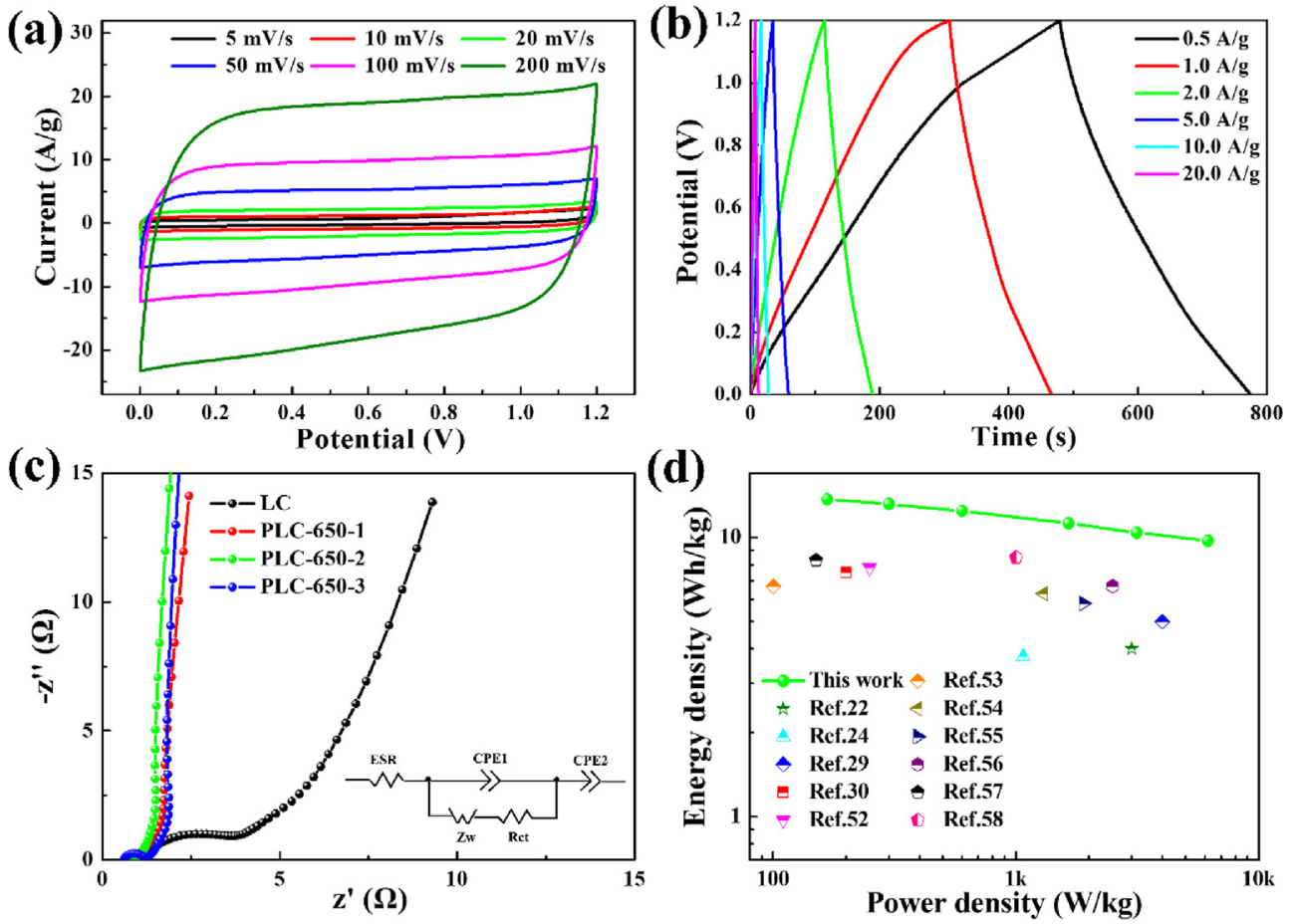

(d)

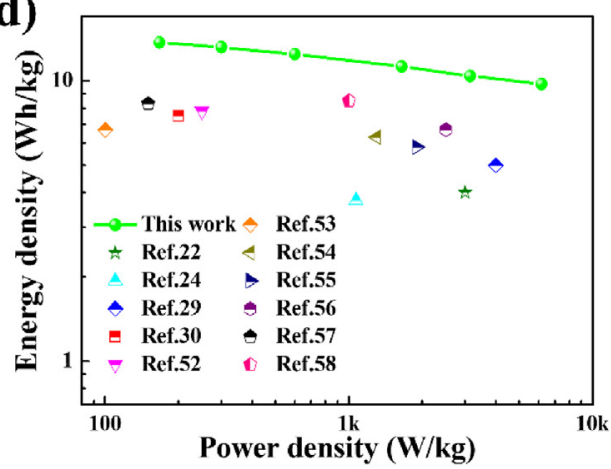

Fig. 6. Electrochemical performance of the symmetric supercapacitors in a two-electrode system in PVA/KOH electrolyte: (a) CV curves at various scan rates and (b) GCD curves at various current densities of PLC650-2 based supercapacitor, (c) Nyquist plots and fitting circuit (inset) of LC, PLC650-1, PLC-650-2, and PLC-650-3 based supercapacitor, (d) Ragone plot of PLC-650-2 based symmetric supercapacitor compared with those of lignin-based or biomass-derived carbons in other literature.

\section{Appendix A. Supplementary data}

Supplementary data to this article can be found online at https:// doi.org/10.1016/j.cej.2019.123721.

\section{References}

[1] G. Yu, X. Xie, L. Pan, Z. Bao, Y. Cui, Nano Energy 2 (2013) 213-234.

[2] P. Simon, Y. Gogotsi, B. Dunn, Science 343 (2014) 1210-1211.

[3] Y. Wang, Y. Song, Y. Xia, Chem. Soc. Rev. 45 (2016) 5925-5950.

[4] Z. Yu, L. Tetard, L. Zhai, J. Thomas, Energy Environ. Sci. 8 (2015) 702-730.

[5] T. Chen, L. Dai, J. Mater. Chem. A 2 (2014) 10756-10775.

[6] Z. Wang, P. Tammela, M. Strømme, L. Nyholm, Adv. Energy Mater. 7 (2017) 1700130.

[7] T. Lv, M. Liu, D. Zhu, L. Gan, T. Chen, Adv. Mater. 30 (2018) e1705489.

[8] C. Wang, D. Wu, H. Wang, Z. Gao, F. Xu, K. Jiang, J. Power Sources 363 (2017) 375-383.

[9] S. Song, F. Ma, G. Wu, D. Ma, W. Geng, J. Wan, J. Mater. Chem. A 3 (2015) 18154-18162.

[10] Y. Chen, X. Zhang, D. Zhang, P. Yu, Y. Ma, Carbon 49 (2011) 573-580.

[11] Y. Xu, Z. Lin, X. Zhong, X. Huang, N.O. Weiss, Y. Huang, X. Duan, Nat. Commun. 5 (2014) 4554.

[12] F. Li, X. Wang, R. Sun, J. Mater. Chem. A 5 (2017) 20643-20650.

[13] Y. Rangom, X. Tang, L.F. Nazar, ACS Nano 9 (2015) 7248-7255.

[14] C. Zhu, T. Liu, F. Qian, T.Y. Han, E.B. Duoss, J.D. Kuntz, C.M. Spadaccini, M.A. Worsley, Y. Li, Nano Lett. 16 (2016) 3448-3456.

[15] C. Liu, Z. Yu, D. Neff, A. Zhamu, B.Z. Jang, Nano Lett. 10 (2010) 4863-4868.

[16] Z. Li, L. Zhang, X. Chen, B. Li, H. Wang, Q. Li, Electrochim. Acta 296 (2019) 8-17.

[17] A. Elmouwahidi, E. Bailón-García, A.F. Pérez-Cadenas, F.J. Maldonado-Hódar, F. Carrasco-Marín, Electrochim. Acta 229 (2017) 219-228.

[18] H. Jin, J. Li, Y. Yuan, J. Wang, J. Lu, S. Wang, Adv. Energy Mater. 8 (2018) 1801007.

[19] J. Wang, P. Nie, B. Ding, S. Dong, X. Hao, H. Dou, X. Zhang, J. Mater. Chem. A 5 (2017) 2411-2428.

[20] S. Chatterjee, T. Saito, ChemSusChem 8 (2015) 3941-3958.

[21] Q. Tang, M. Zhou, Y. Li, X. Qiu, D. Yang, ACS Sustain. Chem. Eng. 6 (2018) 1379-1386.

[22] J.W. Jeon, L. Zhang, J.L. Lutkenhaus, D.D. Laskar, J.P. Lemmon, D. Choi, M.I. Nandasiri, A. Hashmi, J. Xu, R.K. Motkuri, C.A. Fernandez, J. Liu, M.P. Tucker, P.B. McGrail, B. Yang, S.K. Nune, ChemSusChem 8 (2015) 428-432.

[23] N. Guo, M. Li, X. Sun, F. Wang, R. Yang, Green Chem. 19 (2017) 2595-2602.

[24] W. Zhang, H. Lin, Z. Lin, J. Yin, H. Lu, D. Liu, M. Zhao, ChemSusChem 8 (2015) 1114-2122.

[25] Z. Zhao, S. Hao, P. Hao, Y. Sang, A. Manivannan, N. Wu, H. Liu, J. Mater. Chem. A 3 (2015) 15049-15056.

[26] D. Saha, Y. Li, Z. Bi, J. Chen, J.K. Keum, D.K. Hensley, H.A. Grappe, H.M. Meye 3rd, S. Dai, M.P. Paranthaman, A.K. Naskar, Langmuir 30 (2014) 900-910.

[27] L. Zhang, T. You, T. Zhou, X. Zhou, F. Xu, A.C.S. Appl, Mater. Interfaces 8 (2016) 13918-13925.

[28] M. Klose, R. Reinhold, F. Logsch, F. Wolke, J. Linnemann, U. Stoeck, S. Oswald,
M. Uhlemann, J. Balach, J. Markowski, P. Ay, L. Giebeler, ACS Sustain. Chem. Eng. 5 (2017) 4094-4102.

[29] J. Pang, W. Zhang, J. Zhang, G. Cao, M. Han, Y. Yang, Green Chem. 19 (2017) 3916-3926.

[30] J. Pang, W. Zhang, H. Zhang, J. Zhang, H. Zhang, G. Cao, M. Han, Y. Yang, Carbon 132 (2018) 280-293.

[31] W. Zhang, C.L. Yu, L. Chang, W. Zhong, W. Yang, Electrochim. Acta 282 (2018) 642-652.

[32] Y. Chen, G. Zhang, J. Zhang, H. Guo, X. Feng, Y. Chen, J. Mater. Sci. Technol. 34 (2018) 2189-2196.

[33] X. Zheng, J. Luo, W. Lv, D.W. Wang, Q.H. Yang, Adv. Mater. 27 (2015) 5388-5395.

[34] J. Wang, Y. Qian, Y. Deng, D. Liu, H. Li, X. Qiu, Appl. Surf. Sci. 390 (2016) $617-622$.

[35] Y. Zhao, S. Huang, M. Xia, S. Rehman, S. Mu, Z. Kou, Z. Zhang, Z. Chen, F. Gao, Y. Hou, Nano Energy 28 (2016) 346-355.

[36] B. Wang, Y. Wang, Y. Peng, X. Wang, N. Wang, J. Wang, J. Zhao, Chem. Eng. J. 348 (2018) 850-859.

[37] J. Hou, K. Jiang, R. Wei, M. Tahir, X. Wu, M. Shen, X. Wang, C. Cao, A.C.S. Appl, Mater. Interfaces 9 (2017) 30626-30634.

[38] S. Lu, M. Jin, Y. Zhang, Y. Niu, J. Gao, C. Li, Adv. Energy Mater. 8 (2018) 1702545.

[39] S. Biniak, G. Szymański, J. Siedlewski, A. Swiatkowski, Carbon 35 (1997) 1799-1810.

[40] E. Desimoni, G.I. Casella, A.M. Salvi, Carbon 30 (1992) 521-526.

[41] L. Qie, W. Chen, X. Xiong, C. Hu, F. Zou, P. Hu, Y. Huang, Adv. Sci. 2 (2015) 1500195.

[42] J. Liang, Y. Jiao, M. Jaroniec, S.Z. Qiao, Angew. Chem. Int. Ed. 124 (2012) 11664-11668.

[43] L. Zhu, F. Shen, R. Smith Jr, L. Yan, L. Li, X. Qi, Chem. Eng. J. 316 (2017) 770-777.

[44] B. Chang, H. Yin, X. Zhang, S. Zhang, B. Yang, Chem. Eng. J. 312 (2017) 191-203.

[45] J. Shao, M. Song, G. Wu, Y. Zhou, J. Wan, X. Ren, F. Ma, Energy Storage Mater. 13 (2018) 57-65.

[46] X. Fan, C. Yu, J. Yang, Z. Ling, C. Hu, M. Zhang, J. Qiu, Adv. Energy Mater. 5 (2015) 1401761.

[47] D. Kang, Q. Liu, J. Gu, Y. Su, W. Zhang, D. Zhang, ACS Nano 9 (2015) $11225-11233$.

[48] H. Feng, H. Hu, H. Dong, Y. Xiao, Y. Cai, B. Lei, Y. Liu, M. Zheng, J. Power Sources 302 (2016) 164-173.

[49] M. Liu, J. Niu, Z. Zhang, M. Dou, F. Wang, Nano Energy 51 (2018) 366-372.

[50] P. Justin, S.K. Meher, G.R. Rao, J. Phys. Chem. C 114 (2010) 5203-5210.

[51] L. Peng, Z. Fang, Y. Zhu, C. Yan, G. Yu, Adv. Energy Mater. 8 (2018) 1702179.

[52] Y. Huang, L. Peng, Y. Liu, G. Zhao, J.Y. Chen, G. Yu, A.C.S. Appl, Mater. Interfaces 8 (2016) 15205-15215.

[53] Y. Gong, D. Li, C. Luo, Q. Fu, C. Pan, Green Chem. 19 (2017) 4132-4140.

[54] D. Salinas-Torres, R. Ruiz-Rosas, M.J. Valero-Romero, J. Rodríguez- Mirasol, T. Cordero, E. Morallón, D. Cazorla-Amorós, J. Power Sources 326 (2016) 641-651.

[55] W. Zhang, M. Zhao, R. Liu, X. Wang, H. Lin, Colloids Surf. A 484 (2015) 518-527.

[56] Y. Huang, Y. Liu, G. Zhao, J.Y. Chen, J. Mater. Sci. 52 (2017) 478-488.

[57] Z. Hao, J. Cao, Y. Wu, X. Zhao, Q. Zhuang, X. Wang, X. Wei, J. Power Sources 361 (2017) 249-258.

[58] Z. Hao, J. Cao, Y. Dang, Y. Wu, X. Zhao, X. Wei, ACS Sustain. Chem. Eng. 7 (2019) 4037-4046. 